\title{
THE RADIAL BRIGHTNESS DISTRIBUTION OF THE SUN AT 9.4 CM.
}

\author{
F. T. HADDOCK \\ Naval Research Laboratory, Washington, D.C., U.S.A.*
}

The Naval Research Laboratory has supported four eclipse expeditions (in the years 1947, 1950, $195^{2}$ and 1954) under the direction of Dr J. P. Hagen. The principal purpose was to find the variation of the solar microwave radiation during a total optical eclipse of the sun. During the last two eclipses the sun was sufficiently inactive to enable us to derive the centre-to-limb brightness distribution at a wave-length of $9.4 \mathrm{~cm}$., on the assumption that the distribution was circularly symmetrical. Since these two eclipses were of the same optical magnitude (within $0.12 \%$ ) and were measured with the same equipment located at each eclipse near the centreline of mid-totality, it is of interest to compare their results. The 1954 eclipse observation was described in the last paper by Mayer, Sloanaker and Hagen. The 1952 observation has already been described [1].

The 30 June 1954 eclipse occurred at a time of exceptionally low solar activity as evidenced by both optical and radio observations. The unexpectedly high degree of symmetry and smoothness of the eclipse curve described in the following article indicates a regular, unchanging, quiet solar atmosphere. It is unlikely that within the next several decades the sun will again be as quiet during an eclipse.

The 25 February $195^{2}$ eclipse also occurred when there were no visible sunspots. However, a bright calcium plage was seen on each limb near the solar equator. Sudden changes in the observed eclipse curve corresponded in time to the immersion and emersion of these plage regions. No other sudden changes or bright plages were noted. In order to derive an eclipse curve representing the quiet sun it was necessary to subtract the excess radiation from these localized active regions. This was done by attributing differing amounts of excess emission to the two regions until the four sudden changes were smoothed out and the resulting eclipse curve was symmetrical about mid-totality. The amount of excess flux required to do

- Now at the University of Michigan Observatory, Ann Arbor, Michigan, U.S.A. 
this was from 7.5 to $10 \%$ of the total uneclipsed flux. The larger value agrees well with the $12 \%$ excess radiation estimated from the low daily flux level of the undisturbed sun a few days after the eclipse to that on the eclipse day.

Fig. I shows the corrected eclipse curve for $195^{2}$ and the observed eclipse curve for 1954, each with their maximum flux normalized to Ioo. Actually the uneclipsed flux for 1952, after subtraction of the excess flux, was about $15 \%$ greater than the 1954 value. This point will be mentioned later in connexion with the difference between the minimum flux values.

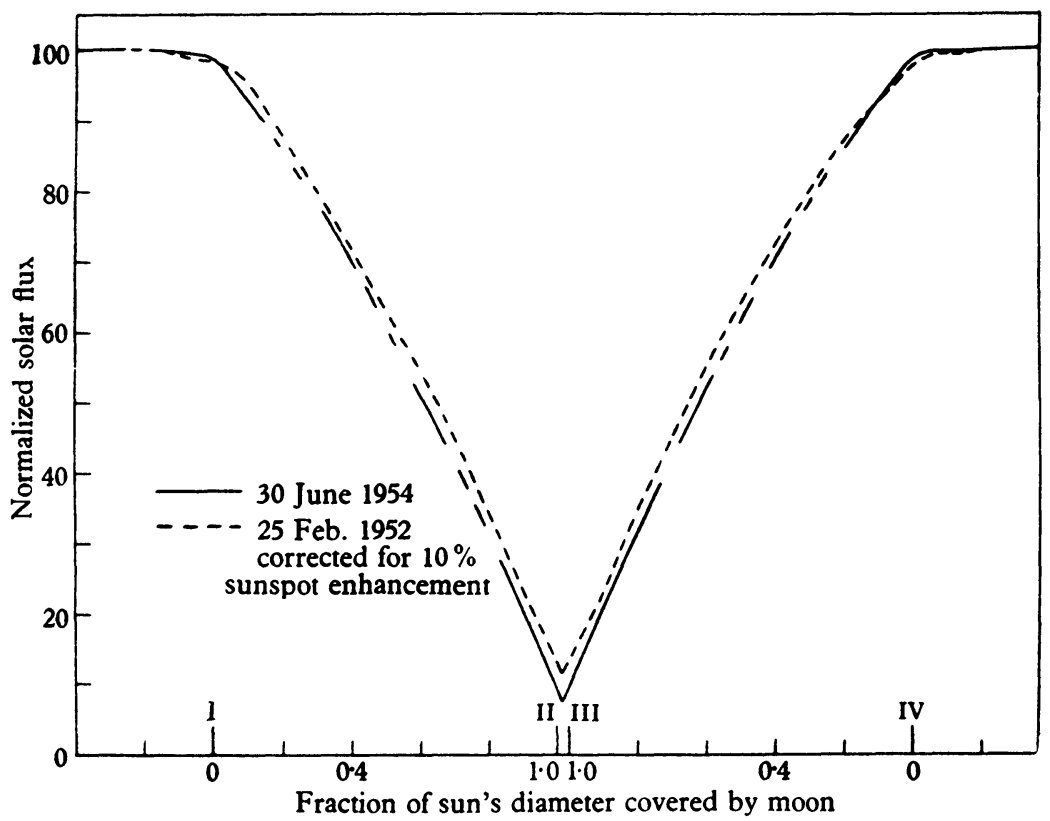

Fig. 1. Eclipse curves at $\lambda 9 * 4 \mathrm{~cm}$. for two eclipses.

The curves agree well in shape, both having fairly straight sides and a sharp V-shaped minimum. The disagreement in the regions of $I$ and IV contact should be expected because the $195^{2}$ curve was corrected for excess emission in those regions and, furthermore, the curves must cross to be consistent with their minimum values. The $195^{2} \mathrm{~V}$-shaped minimum was felt to be artificial when it was first derived but it was found difficult to deviate far from it and still produce a symmetrical eclipse curve. The fact that the 1954 observed eclipse curve displayed a sharp V-shaped minimum supports the validity of the corrections made on the $195^{2}$ curve.

It is impossible to derive a two-dimensional projected brightness distri- 
bution of the sun from a single one-dimensional eclipse curve (or a single fanbeam scan) without arbitrarily restricting the form of the distribution. For simplicity it is commonly assumed that the sun's atmosphere is spherically symmetrical, producing circular radio isophotes. However, it has been known for several years that elliptical isophotes are better representative of the sun at metre wave-lengths, and recently Christiansen and Warburton [2] have derived a two-dimensional brightness distribution of the quiet sun at $2 \mathrm{I}-\mathrm{cm}$. wave-length which has quadrant symmetry with pronounced limb brightening in the equatorial regions changing smoothly to limb darkening at the poles. The $9.4 \mathrm{~cm}$. brightness distribution should be similar to this, although its deviation from circular symmetry may be less since the coronal contribution to the brightness is appreciably less.

Radial brightness distributions with circular symmetry have been found giving eclipse curves which agree with the observed curves to within I $\%$ of the sun's uneclipsed flux. These distributions are not unique, however, even with the assumption of circular symmetry, because there exists a set of radial brightness functions which are not visible in the eclipse curve. That is, they can be added to a given radial brightness distribution without changing the associated eclipse curve. These 'invisible' radial functions can be represented by a linear combination of the zero-order Bessel functions, centred on the sun, which have a maximum or a minimum value at the radial distance which equals the moon's radius (unpublished work by S. Ament). We do not have a good method for eliminating these fictitious functions hidden in derived distributions, although their effect can be reduced by smoothing the data. Their presence accounts for the variation of shape in the set of distributions which were found to satisfy the I952 eclipse data. The distribution shown in Fig. 2 was chosen from this set because it resembled in shape the theoretical distributions. The 1954 data are more precise and with simple smoothing produced the distribution shown in Fig. 2. It closely resembles published theoretical distributions [3]. Both distributions in Fig. 2 have a peak brightness of about twice the central brightness, with the peak located just beyond the optical limb of the sun.

An indication of the degree of deviation from circular symmetry in the actual brightness distribution can be obtained in the following way. The upper part of the eclipse curve (lunar positions for which the centre of the solar disk is uneclipsed) completely determines the lower part of the eclipse curve if the brightness distribution has circular symmetry. On the other hand, if for example the distribution has a brighter limb at the equator than at the poles, and the track of the moon's centre over the disk 
does not make an angle with the sun's equator close to $45^{\circ}$, then the upper part of the eclipse curve would predict, on the basis of circular symmetry, a lower part in discord with observation. For the two curves in Fig. I this discordance was comparable with the observational uncertainties from which it was estimated that only a small percentage of solar flux at $9.4 \mathrm{~cm}$. arises from a non-symmetrical component in the actual brightness distribution. Therefore the distributions shown in Fig. 2 are believed to be reasonable representations of the brightness distribution of the quiet sun on the two eclipse days. They are in general agreement with theoretical models of the quiet sun. The fact that the bright limb occurs outside the

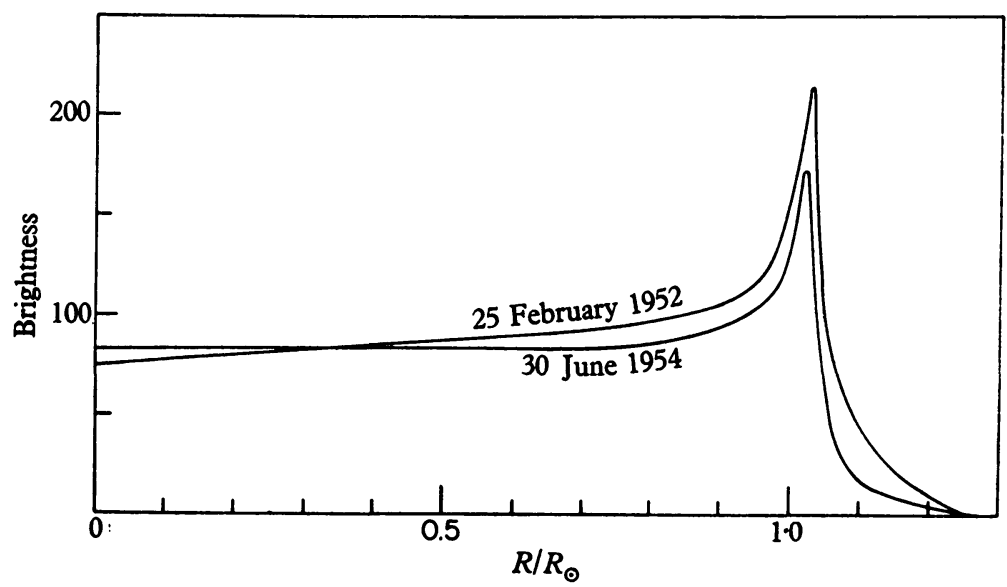

Fig. 2. Radial brightness distributions at $\lambda 9 \cdot 4 \mathrm{~cm}$.

optical disk is a significant point of agreement with theory since there are notable exceptions to this in published distributions, obtained with fanbeam scans, at wave-lengths of $10.3 \mathrm{~cm}$. [4] and $21 \mathrm{~cm}$. [2] for example.

The principal long-range value of the eclipse observations will undoubtedly lie in the eclipse curves themselves because they can be used as standards against which any future brightness distributions of the quiet sun near sunspot minimum can be tested by generating artificial eclipse curves for a magnitude of $\mathrm{I} \cdot 036$. In fact, any model of the sun's atmosphere from which a $9.4 \mathrm{~cm}$. brightness distribution could be derived could be tested in the same way.

The minimum flux value, measured at mid-eclipse, is of special interest because an eclipse of magnitude $\mathrm{I} \cdot 036$ completely occults the chromosphere and the exposed part of the corona is both optically thin and nonrefracting for wave-lengths shorter than about $30 \mathrm{~cm}$. Therefore, if the 
radiation is thermal, the minimum flux value is the same at these snort wave-lengths.

It is estimated that the mid-eclipse flux density for the quiet corona on 25 February 1952 was 9.4 units (where one unit equals $10^{-22}$ w.m..$^{-2}$ $(\mathrm{c} . / \mathrm{s} .)^{-1}$ ) and $5^{\cdot I}$ units on $3^{\circ}$ June 1954. Using the Allen values for electron densities and an isothermal corona at $10^{6 \circ}$ it can be shown that one-third (or 13.7 units of flux density) of the total coronal radiation (of 53.5 units) is uneclipsed at mid-eclipse (magnitude of $\mathrm{I} \cdot{ }^{\circ} 36$ ) for all wavelengths shorter than about $30 \mathrm{~cm}$. On the basis of this model the 4.3 unit decrease of mid-eclipse flux requires a 12.9 unit decrease in total coronal flux. The actual drop in the total solar flux was only ro.6 units. Thus the mid-eclipse flux decrease from 1952 to 1954 was greater in proportion to the total flux decrease than expected from the Allen model. A plausible explanation for this is that the emission parameter $\left(N_{e}^{2} . T_{e}^{-1 / 2}\right)$, decreased a greater percentage in the outer corona than in the inner, thereby decreasing the fraction of uneclipsed flux at mid-eclipse. This greater change at greater heights as sunspot minimum is approached agrees with the changes in electron densities given by van de Hulst [3]. Reasonably expected changes in temperature would have a negligible effect in this situation.

It is of interest to compare the observed mid-eclipse flux densities with theoretical values. Van de Hulst [5] has also pointed out that the radio opacity of the corona should decrease by a factor of 3 from the sunspot maximum to minimum. Since the Allen model applies to the sunspot maximum the calculated mid-eclipse flux density would then decrease from 13.7 to 4.5 units at the minimum. This is in substantial agreement with the observed values of 9.4 in $195^{2}$ and $5^{.} .1$ in 1954 .

In 1947 , at a sunspot maximum, the N.R.L. eclipse expedition measured at $3.2 \mathrm{~cm}$. a flux density at mid-totality during an eclipse of nearly the same magnitude $(\mathrm{I} \cdot 038)$ of about $\mathrm{I} 5$ units. Since emission from the moon can only account for 3 or 4 units of this value, we again have reasonable agreement with the calculated flux density.

For various reasons, such as the corona being optically thin and nonrefracting and the precise geometry at mid-eclipse, it appears that this type of measurement is a good test for a model of the corona; it is also sensitive to the height scale of the model. For example, with the $9.4 \mathrm{~cm}$. equipment it would be possible to detect the change in flux caused by a change in the moon's radius of less than $I^{\prime}$ arc at mid-eclipse. 


\section{REFERENCES}

[1] Haddock, F. T. J. Geophys. Res. 59, 174, 1954.

[2] Christiansen, W. N. and Warburton, J. A. Observatory, 75, 9, 1955.

[3] Hagen, J. P. Ap. J. 113, 547, 195 I.

[4] Covington, A. E. and Broten, N. W. Ap. J. 119, 585, 1954.

[5] van de Hulst, H. C. Nature, 163, 24, I949.

\section{Discussion}

Hey: Observations of partial eclipses can be very useful in indicating deviations from circular asymmetry. We observed the eclipse of 30 June 1954 at Io $\mathrm{cm}$. wave-length from a site where the eclipse was partial and we found an indication that the intensity distribution across the solar disk must have greater equatorial than polar extent. Is there any such indication in your data?

Haddock: If $5 \%$ of the total flux were distributed in a double sine wave around the limb of the sun a slightly better fit would be obtained. 